\title{
A second-order autocorrelator for single-shot measurement of femtosecond laser pulse durations
}

\author{
M RAGHURAMAIAH, A K SHARMA, P A NAIK, P D GUPTA and \\ R A GANEEV*
}

Plasma Radiation Section, Centre for Advanced Technology, Indore 452 013, India *On visit from NPO Akadempribor, Uzbek Academy of Sciences, Tashkent

700 143, Uzbekistan

e-mail: raghuram@cat.ernet.in

MS received 2 May 2001; revised 4 September 2001

\begin{abstract}
A second-order autocorrelator for single-shot measurement of ultrashort laser pulse durations has been set up. It is based on recording the spatial profile of non-collinear phase-matched second harmonic generation in a KDP crystal using a CCD camera-framegrabber combination. Performance of the system is described from measurement of 250 femtosecond transform-limited laser pulses from a passively mode-locked, diode pumped $\mathrm{Nd}$ :glass laser. It can also be used for measurement of picosecond laser pulses in the multi-shot scanning mode.
\end{abstract}

Keywords. Autocorrelation; femtosecond laser pulses; Nd:glass laser; second harmonic radiation.

\section{Introduction}

Recently there has been a great upsurge of interest in using femtosecond laser pulses for studies of ultrafast phenomena (Brabec \& Krausz 2000). Laser pulses of duration ranging from a few femtoseconds to a few hundred femtoseconds are reliably produced through different mode-locking techniques (Liu et al 1992; Bilinsky et al 1998). Amplification of these pulses using a chirped pulse amplification scheme (Strickland \& Mourou 1985) has enabled generation of compact multi-terawatt laser systems, which are increasingly being used for a variety of investigations on laser-matter interaction in high field regimes (Mourou et al 1998; Kruer 2000). Temporal characterization of these laser pulses is an important aspect of such investigations to understand the dynamics of the processes involved. Since electronic devices and even streak cameras are too slow for temporal measurements of femtosecond laser pulses, intensity correlation techniques (Ippen \& Shank 1984) are employed for this purpose. While measurement of second-order autocorrelation facilitates an estimation of laser pulse duration (Janszky et al 1977; Ishida et al 1985; Salin et al 1987; Braun et al 1995; Curley et al 1995; Ganeev et al 1995), higher order correlations are necessary to obtain the temporal shape as well (Luan et al 1993; Feurer et al 1998; Osvay et al 1999). Further, spectrally resolved autocorrelation in conjunction with sophisticated algorithms (for example, frequency 
resolved optical gating, FROG techniques) have been reported to obtain information on both the amplitude and phase of ultrashort laser pulses (Paye et al 1993; Walmsley \& Wong 1996).

Knowledge of laser pulse duration is the foremost requirement in most of the investigations on laser-matter interaction involving ultrashort laser pulses. As stated earlier, this can be accomplished through a second-order autocorrelation measurement. Although such autocorrelators are commercially available, they are quite expensive. Further, design of these autocorrelators depends on the range of laser pulse duration and whether the measurement is to be carried out in a single-shot operation (Janszky et al 1977; Ishida et al 1985; Salin et al 1987; Ganeev et al 1995) or in a multi-pulse scanning mode (Ippen \& Shank 1984; Braun et al 1995; Curley et al 1995). It is therefore desirable to set up these devices in the laboratory to have flexibility in their operation and selection of the parameter range. In this paper, we report on indigenous development of a second order autocorrelator for single-shot measurements of pulse durations of ultrashort laser pulses. It is based on recording the spatial profile of non-collinear phase-matched second-harmonic generation in a KDP crystal using a CCD camera-framegrabber combination. Design parameters and experimental set up are described. The autocorrelator has been used for measurement of 250 femtosecond transformlimited laser pulses from a passively mode-locked, diode-pumped Nd:glass laser. By a simple change of detection system, the autocorrelator can be used for measurement of picosecond laser pulses in the multi-shot scanning mode. Measurements of pulse duration and peak to background intensity contrast ratio of $25 \mathrm{ps}$ laser pulses from an active-passive mode-locked Nd:YLF laser carried out in this mode are also presented.

\section{Principle}

The second order autocorrelation technique basically involves splitting a laser beam into two beams of equal intensity and overlapping them in a nonlinear crystal to produce second harmonic $(\mathrm{SH})$ radiation. Since the intensity of $\mathrm{SH}$ is proportional to the product of the intensities $I(t)$ of the two beams, its measurement as a function of relative time delay $\left(t_{d}\right)$ between them would provide the autocorrelation function $A\left(t_{d}\right)$ given by

$$
A\left(t_{d}\right)=\int_{-\propto}^{\propto} I(t) I\left(t-t_{d}\right) \mathrm{d} t .
$$

The pulse duration, $t_{p}$ (FWHM), of the laser pulse can be deduced from the FWHM of the autocorrelation function, $A\left(t_{d}\right)$, provided the pulse shape is assumed. The autocorrelation function can be measured in a multi-shot scanning operation by introducing different delays in the two laser pulses, and the same is used for pulse duration measurement in the range of tens of picoseconds to a few hundred picoseconds.

In contrast to the above approach, second order autocorrelation for laser pulses of duration tens of femtoseconds to a few picoseconds can be recorded in a single shot due to their small spatial extent ( $=c t_{p}$ e.g. $30 \mu \mathrm{m}$ for $100 \mathrm{fs}$ pulse). Here again, the input beam is split into two beams, which are then made to cross each other at a small angle inside a non-linear crystal as shown in figure 1. Provided the beam waist in the overlap region is much larger than the pulse length, $c t_{p}$, the two beams can be made to overlap temporally and spatially inside the crystal. In such a geometry, at each overlapping point, the SH signal generated is proportional to the product of local intensity in each path, and the direction of emission of the signal is along the 


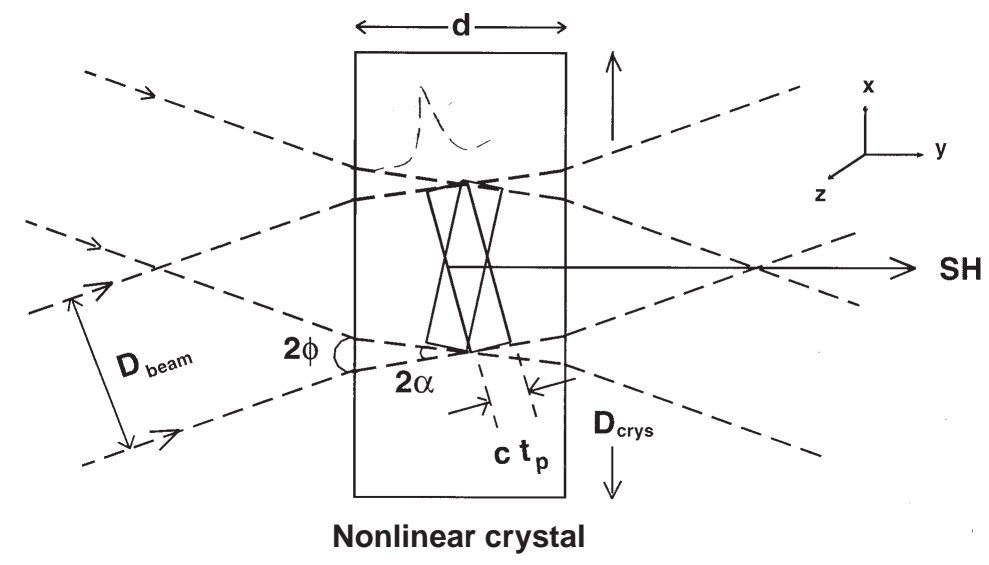

Figure 1. Schematic of achieving single-shot, second-order autocorrelation of femtosecond laser pulses from spatial intensity profile of second-harmonic beam generated in a nonlinear crystal.

bisector of the crossover angle. For any line parallel to the bisector, the generated SH signal represents the time-integrated intensity product. Since the beams cross at an angle, different lines parallel to the bisector correspond to overlap for different time intervals. The central line (bisector of the overlap region) corresponds to a full temporal overlap of the two pulses. In this way, the autocorrelation function is transformed into a spatial intensity distribution (along the $x$ direction) of SH emission which can be recorded with a CCD camera. FWHM $(\Delta x)$ of the spatial intensity distribution is related to the laser pulse duration (Ishida et al 1985) as,

$$
t_{p}=(k \Delta x \sin \phi) / c,
$$

where $\phi$ is the half angle between the two beams (outside the crystal) and $k$ is a numerical factor governed by the laser pulse shape. It can be easily shown that for gaussian and $\operatorname{sech}^{2}$ pulse shapes, $k$ is $\sqrt{ } 2$ and $\sim 1.3$ respectively.

\section{Design considerations}

Physical size of the nonlinear crystal and its orientation, diameter of the two overlapping beams, and the angle between them, are important parameters governing the design of the single-shot autocorrelator. For non-collinear geometry of the overlapping beams, the phase matching condition is

$$
n_{o}(\omega) \cos \alpha=n_{e}(2 \omega),
$$

where $n_{o}(\omega)$ and $n_{e}(2 \omega)$ denote the ordinary and extraordinary refractive indices at fundamental and second-harmonic frequency respectively, and $2 \alpha$ is the crossover angle of the beams inside the crystal. For a KDP crystal used for second harmonic generation of Nd:glass laser pulses, the maximum value of $2 \alpha$ is $\simeq 20^{\circ}$. Thus the maximum crossover angle $2 \phi$ (outside the crystal) of the two beams can be $30^{\circ}$.

Conditions on the beam diameter and thickness of the crystal can be obtained on referring to figure 1. A complete temporal overlap of the two pulses travelling as two beams of diameter $D_{\text {beam }}$ would occur, if

$$
D_{\text {beam }} \tan \phi \geq c t_{p} \text {. }
$$


Further, temporal overlap of the two beams would occur in a crystal of thickness $d$, if

$$
d \cos \alpha \geq(c / n) t_{p} .
$$

Finally the aperture of the crystal, $D_{\text {crys }}$, should be large enough to accommodate the two incident beams i.e.

$$
D_{\text {crys }} \geq\left(D_{\text {beam }} / \cos \phi\right)+(d \tan \alpha)
$$

It follows from (3) to (6) that by varying $D_{\text {beam }}$ and $\phi$, one can make single shot measurements of pulse duration over a wide temporal range using KDP crystals cut for non-collinear phase matching.

For a type I KDP crystal, the phase matching angle, $\theta$, is $41^{\circ}$ for the collinear geometry. In the non-collinear geometry (Ganeev et al 1995), the phase matching angle is given by

$$
\sin ^{2} \theta=\left[\left(n_{o}^{2}(2 \omega) \sec ^{2} \alpha / n_{o}^{2}(\omega)\right)-1\right]\left[\left(n_{o}^{2}(2 \omega) / n_{e}^{2}(2 \omega)\right)-1\right]^{-1} .
$$

It is seen from the above equation that phase matching angle in a non-collinear geometry is larger than that for collinear geometry. Ideally the crystal face should be cut at the noncollinear phase-matching angle. However, for small values of $\phi\left(\leq 5^{\circ}\right)$, it is practical to orient the crystal cut for collinear phase matching by a small angle to meet the requirement of (7).

In our autocorrelator design, we have taken $\phi=2^{\circ}$ so that the phase-matching angle for the non-collinear geometry is $\theta \approx 42^{\circ}$. The KDP crystal (type I) is therefore required to be oriented at a small angle of $1^{\circ}$ with respect to the collinear phase-matching geometry. Further, for this choice, the requirement on the beam diameter and crystal thickness from (4) and (5), turn out to be $D_{\text {beam }(\mathrm{mm})} \geq 8.6 \times t_{p}(\mathrm{ps})$, and $d(\mathrm{~mm}) \geq 0.2 \times t_{p}(\mathrm{ps})$. These conditions on beam diameter and crystal size are quite easy to meet.

As mentioned earlier, longer pulse durations can also be measured in a single shot by using larger values of crossover angle, $2 \phi$. For the maximum value of $\phi=15^{\circ}$ for KDP crystal, the requirement on crystal size and thickness would be $D_{\text {crys }(\mathrm{mm})} \geq 1.16 \times t_{p}(\mathrm{ps})$ and $d(\mathrm{~mm}) \geq 0.2 \times t_{p}(\mathrm{ps})$. Therefore, the limit on the single-shot measurement of long duration pulses would come from the availability of large size crystals of good quality. On the other hand, the limit on short-pulse duration measurements arises from the group velocity dispersion (GVD) effects in the crystal and spatial resolution of the imaging system. In the case of ultrashort pulses, due to the broadband spectra of the interacting pulses, pulse broadening occurs due to GVD during propagation through the crystal given by

$$
\Delta t_{p}=(\lambda d / c)(\Delta \lambda)\left(\mathrm{d}^{2} n / \mathrm{d} \lambda^{2}\right) .
$$

For a KDP crystal and $\lambda=1054 \mathrm{~nm}$, pulse broadening factor is $0.02 \mathrm{fs} / \mathrm{nm}$-mm. Moreover, the group velocity mismatch, $V_{\text {mismatch }}(9 \mathrm{fs} / \mathrm{mm})$ between the fundamental beam and second harmonic radiation would require the thickness of the crystal to be smaller than the walk-off length $\left(=t_{p} / V_{\text {mismatch }}\right)$. Thus the GVD effects become significant while dealing with pulse durations of $10-20 \mathrm{fs}$ or smaller.

\section{Experimental set-up}

Experimental set-up for single-shot measurement is shown in figure 2. The laser beam, for which pulse duration measurement is to be carried out, is split into two equal intensity beams 


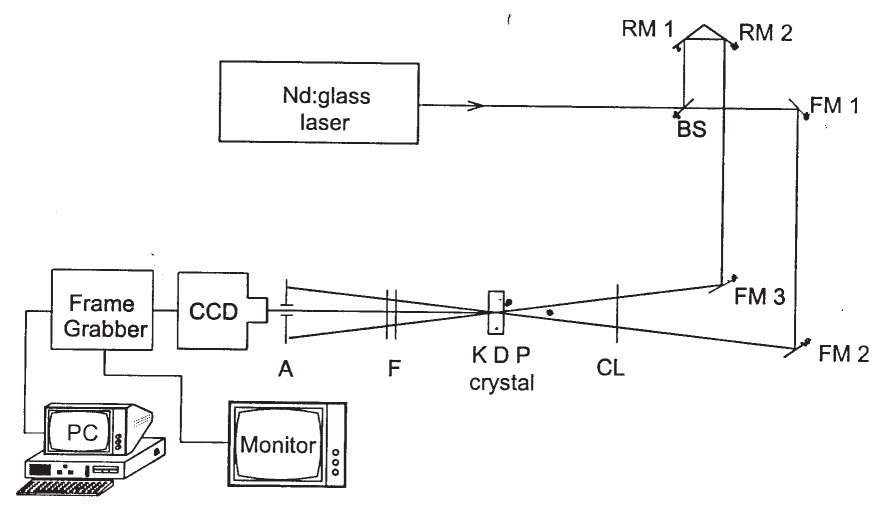

Figure 2. Experimental set-up of single-shot autocorrelator. RM: retro mirror, FM: folding mirror, BS: beam splitter, CL: cylindrical lens, F: filters, A: aperture.

using a beam splitter, BS. In order to ensure a complete temporal overlap of the two beams in the non-collinear geometry of SH generation in the KDP crystal (figure 1), they should have travelled equal path lengths. This is accomplished by keeping one of the path lengths fixed and varying the other. Variable beam path is achieved by using two gold-coated mirrors, RM1 and RM2 ( $45^{\circ}$ incidence, $100 \%$ reflectivity), in a retro-mirror configuration and rigidly mounting them on a translational stage with an attached micrometer. The second beam of fixed path length is folded through two $100 \%$ reflectivity mirrors, FM1 and FM2. The two beams are then line-focused into the type I $\left(\theta=41^{\circ}\right) \mathrm{KDP}$ crystal $(20 \mathrm{~mm} \times 20 \mathrm{~mm} \times 6 \mathrm{~mm}$ thick) along the $z$-direction (non-autocorrelation direction) using an $f / 5$ cylindrical lens (CL) of $300 \mathrm{~mm}$ focal length. The two beams make a crossover angle $(2 \phi)$ of $4^{\circ}$. Focussing through cylindrical lens helps to increase the intensity of SH radiation. The resultant autocorrelated SH signal is recorded with a $1 / 2$ inch format CCD camera $(512 \times 256$ pixels each of $11.8 \mu \mathrm{m} \times 8.3 \mu \mathrm{m})$ connected to a framegrabber card-PC combination. An iris is placed between the KDP crystal and the CCD camera to prevent the two fundamental beams as well as their individually generated SH radiations from entering the detector. KG-3 filters are used to block any scattered fundamental radiation from being detected, and neutral density filters $(\mathrm{F})$ are placed to control the intensity level of $\mathrm{SH}$ radiation on the $\mathrm{CCD}$ camera.

The above set-up can be easily changed to multi-shot scanning mode for measurement of longer duration pulses by replacing the CCD camera-framegrabber system with a photomultiplier detector and introducing a relative time delay between the two beams through the translational stage mounted with mirrors, RM1 and RM2. A high gain photomultiplier (Philips 2232) is used to detect SH radiation signals. High dynamic range of this photomultiplier coupled with calibrated filters allows measurement of peak to background intensity contrast ratio of the laser pulse in this scanning mode.

\section{Results and discussion}

The above autocorrelator was used to measure pulse durations of output laser pulses from a CW diode-pumped, passively mode-locked Nd:glass laser system (GLX-200, M/s Time Bandwidth products) in the single-shot mode, and output laser pulses of an active-passive mode-locked Nd:YLF laser system (Quantel make) in the multi-shot scanning mode. The former laser system uses a semiconductor saturable mirror for mode-locking and provides 
(a)

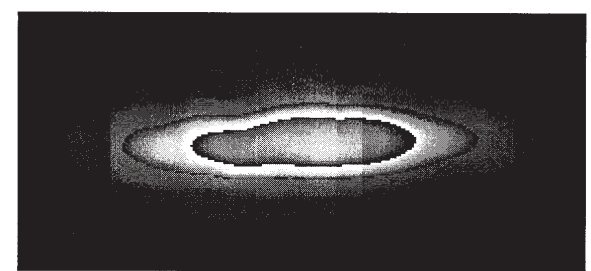

(b)

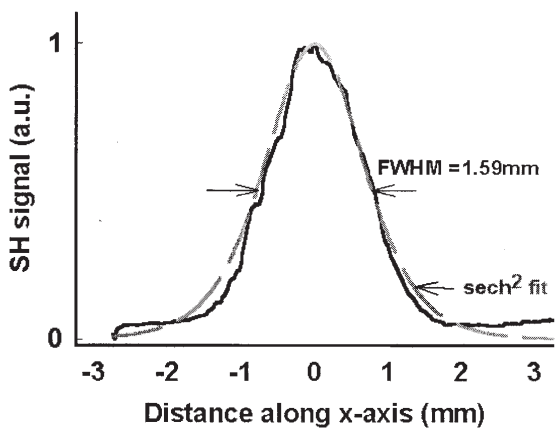

Figure 3. (a) CCD image of the $\mathrm{SH}$ signal (autocorrelation) generated in the KDP crystal. (b) Spatial intensity profile ( $x$-direction) of the SH beam. FWHM of the distribution $(1.59 \mathrm{~mm})$ corresponds to a laser pulse duration of $250 \mathrm{fs}$ (taking correction factor for input intensity variation across the input beam diameter). Dotted line shows the calculated autocorrelation function for a $\operatorname{sech}^{2}$ shaped laser pulse of $250 \mathrm{fs}$ (FWHM) duration.

transform-limited femtosecond laser pulses $(\lambda=1054 \mathrm{~nm})$ at a repetition rate of $100 \mathrm{MHz}$. The other laser system delivers $\sim 25 \mathrm{ps}$ (FWHM) laser pulses at a repetition rate of $10 \mathrm{~Hz}$. Figure 3a shows the image of $\mathrm{SH}$ radiation from the KDP crystal corresponding to the singleshot operation of the autocorrelator for femtosecond laser pulses from the GLX-200 system. This image was analysed using PROMISE software developed at our Centre (Vora et al 1994). A spatial intensity profile scan in the $x$-direction (autocorrelation direction) for the above image is shown in figure $3 \mathrm{~b}$. FWHM of this autocorrelation function, $\Delta x$, is measured to be $1.59 \mathrm{~mm}$. The value of the laser pulse duration can be estimated from this, assuming the laser pulse shape.

The GLX-200 laser system uses a prism pair, providing negative dispersion, to compensate for the positive dispersion due to the active medium in its cavity. The only stable solution, for evolution of a pulse in such a cavity having self-phase modulation and net negative dispersion, comes out to be $\operatorname{sech}^{2}$ in nature (Krausz et al 1992). Therefore, we take the $\operatorname{sech}^{2}$ form for the laser pulse shape (as also specified in the manufacturer's data sheet) i.e.

$$
I(t)=4 /\left(e^{t / \tau}+e^{-t / \tau}\right)^{2},
$$

for which the FWHM pulse duration,

$$
t_{p}=2 \tau \ln (1+\sqrt{2}) .
$$

Autocorrelation function due to overlap of two such pulses for the geometry shown in figure 1 can be calculated to be

$$
I_{\mathrm{SH}}(x)=A_{0}\left\{(X \operatorname{coth} X-1) / \sinh ^{2} X\right\},
$$

where

$$
X=2 x \sin \phi / c \tau .
$$


FWHM $(\Delta x)$ of the autocorrelation function, (11), is

$$
\Delta x=(1.36 c \tau) / \sin \phi .
$$

Using (10), we get

$$
\Delta x=\left(0.77 c t_{p}\right) / \sin \phi .
$$

Thus for the autocorrelation function represented by the SH spatial profile of figure $3 \mathrm{~b}$ having $\Delta x=1.59 \mathrm{~mm}$, the laser pulse duration comes to $\sim 240 \mathrm{fs}$, assuming a uniform intensity profile for the input beam. However, in practical cases, the intensity may vary across the input beam diameter which may lead to slight modification in the estimated value of pulse duration. Corresponding to the above stated FWHM value of $\Delta x=1.59 \mathrm{~mm}$, the variation in intensity for a gaussian profile of $9 \mathrm{~mm}$ beam diameter ( $1 / e^{2}$ points), as in our case, is $6 \%$. A correction factor of $\approx 1.05$ is estimated for the actual pulse duration by comparing the computed autocorrelation function corresponding to a Gaussian beam of $9 \mathrm{~mm}$ beam diameter with the measured autocorrelation function (figure $3 \mathrm{~b}$ ). Thus the laser pulse duration in the above measurements becomes $\approx 250 \mathrm{fs}$. Autocorrelation function, (11), calculated for a laser pulse of sech pulse shape and a FWHM pulse duration of $250 \mathrm{fs}$ is shown as a dotted line in figure $3 \mathrm{~b}$, and a close match is observed between the experimentally observed and theoretically calculated autocorrelator functions. The measured pulse duration of $250 \mathrm{fs}$ is also in good agreement with the transform-limited nature of the output laser pulse. The spectral width $(\Delta \lambda)$ of the laser pulse was monitored using a monochromator and measured to be $\approx 5.2 \mathrm{~nm}(\mathrm{FWHM})$. This provides a value of the time-bandwidth product $(\Delta t \Delta v)$ as 0.351 , which is close to the theoretical value of 0.315 expected for a sech ${ }^{2}$ pulse shape, indicating that the laser pulses are essentially free from frequency chirp.

Next, operation of the aucorrelator in multi-shot scanning mode was tested using longer laser pulses $(\mathrm{FWHM} \approx 25 \mathrm{ps}$ ) from the Nd:YLF active-passive mode-locked oscillator. In the scanning mode, the SH signal is maximum when the two laser pulses coincide in time, and it decreases as their relative time delay is increased. The plot of SH signal as a function of time delay is shown in figure 4. FWHM, $\left(\Delta t_{d}\right)$, of this autocorrelation function was observed to be 36.6 ps. The FWHM duration of the laser pulse can be obtained from the FWHM of the autocorrelation function by making an assumption on the pulse shape. As per the technical data sheets supplied by the manufacturer for the laser system used, these laser pulses have a gaussian shape i.e.

$$
I(t)=I_{0} \exp \left(-2 t^{2} / t_{0}^{2}\right),
$$

for which FWHM pulse duration,

$$
t_{p}=(2 \ln 2)^{1 / 2} t_{0} .
$$

The autocorrelation function, $I_{\mathrm{SH}}\left(t_{d}\right)$ for this pulse in the scanning mode can be calculated to be

$$
I_{\mathrm{SH}}\left(t_{d}\right)=k_{0} \exp \left(-t_{d}^{2} / t_{0}^{2}\right) .
$$

The FWHM of the above autocorrelation function is then

$$
\Delta t_{d}=2(\ln 2)^{1 / 2} t_{0} .
$$




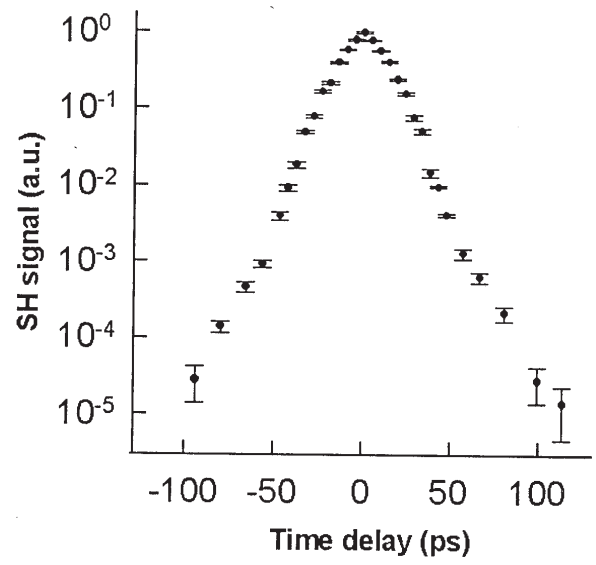

Figure 4. Variation of the SH signal as a function of relative time delay between the two laser pulses in the multi-shot scanning mode.

Using (15), we get

$$
\Delta t_{d}=\sqrt{2} t_{p}
$$

Thus for the present case, the laser pulse duration should be $\approx 26 \mathrm{ps}$. This is in good agreement with the value of 25 ps observed using an S-1 optical streak camera.

An interesting aspect of the scanning mode operation of this autocorrelator is that it can provide an estimate of peak to background intensity contrast ratio of the laser pulses. For this purpose, the SH signal was measured for longer time delays as shown in figure 4 . It is noted that the ratio of SH intensity at no delay (i.e. peak) to that at a time 80 ps prior to the peak is $\approx 10^{4}$. The maximum value of the peak to background intensity contrast ratio in the present experiment was $\approx 7 \times 10^{4}$, limited by the detection system. Thus the peak to background contrast ratio of the laser pulses was higher than this number.

\section{Conclusions}

We have described the design and operation of a simple, second-order autocorrelator based on second-harmonic generation in a KDP crystal for pulse-duration measurement of ultrashort laser pulses. It measures pulse durations of femtosecond laser pulses in a single shot, and longer laser pulses (tens to hundreds of picoseconds) in a multi-shot scanning mode operation. The performance of this system was tested with a diode-pumped Nd:glass laser system providing transform-limited $250 \mathrm{fs}$ (FWHM) laser pulses, and an active-passive mode-locked $\mathrm{Nd}$ :YLF laser providing $25 \mathrm{ps}$ (FWHM) laser pulses. In the latter case, it was possible to measure the peak to background intensity contrast ratio of the laser pulses.

\section{References}

Bilinsky I P, Fujimoto J G, Walpole J N, Missaggia L J 1998 Semiconductor-doped-silica saturableabsorber films for solid-state laser modelocking. Opt. Lett. 23: 1766-1768

Brabec T, Krausz F 2000 Intense few-cycle laser fields: Frontiers of nonlinear optics. Rev. Mod. Phys. 72: $545-591$

Braun A, Rudd J V, Cheng H, Mourou G, Kopf D, Jung I D, Weingarten K J, Keller U 1995 Characterization of short pulse oscillators by means of a high dynamic range autocorrelation measurement. Opt. Lett. 20: 1889-1891 
Curley P F, Darpentigny G, Cheriaux G, Chambaret J P, Antonetti A 1995 High dynamic range autocorrelation studies of a femtosecond Ti:Sapphire oscillator and its relevance to the optimization of chirped pulse amplification systems. Opt. Commun. 120: 71-77

Feurer T, Niedermeier S, Sauerbrey R 1998 Measuring the temporal intensity of ultrashort laser pulses by triple correlation. Appl. Phys. B66: 163-168

Ganeev R A, Ganikhanov F Sh, Gorelik I G, Dakhin A A, Kunin D G, Usmanov T, Zinoviev A V 1995 Laser pulse duration measurements in the range of 0.2 to 50 picosecond. Opt. Commun. 114: 432-434

Ippen E P, Shank C V 1984 Techniques for measurement. UItrashort light pulses: picosecond techniques and applications (ed.) S L Shapiro (New York: Springer Verlag) pp 83-122

Ishida Y, Yajima T, Watanabe A 1985 A simple monitoring system for single subpicosecond laser pulses using an SH spatial autocorrelation method and a CCD image sensor. Opt. Commun. 56: $57-60$

Janszky J, Corradi G, Gyuzalian R N 1977 On a possibility of analyzing the temporal characteristics of short light pulses. Opt. Commun. 23: 293-297

Krausz F, Fermann M E, Brabec T, Curley P F, Hofer M, Ober M H, Spielmann C, Wintner E, Schmidt A J 1992 Femtosecond solid-state lasers. IEEE J. Quantum Electron. QE-28: 2097-2122

Kruer W L 2000 Interaction of plasmas with intense lasers. Phys. Plasmas 7: 2270-2278

Liu K X, Flood C J, Walker D R, van Driel H M 1992 Kerr lens modelocking of a diode pumped Nd:YAG laser. Opt. Lett. 17: 1361-1363

Luan S, Hutchinson M H R, Smith R A, Zhou F 1993 High dynamic range third-order autocorrelation measurement of picosecond laser pulse shapes. Meas. Sci. Technol. 4: 1426-1429

Mourou G A, Barty C P J, Perry M D 1998 Ultrahigh intensity lasers: Physics of the extreme on a table top. Phys. Today 51: 22-28

Osvay K, Ross I N, Lister J M D, Hooker C J 1999 High dynamic range measurement of temporal shape and contrast of ultrashort UV pulses. Appl. Phys. B69: 19-23

Paye J, Ramaswamy M, Fujimoto J G, Ippen E P 1993 Measurement of the amplitude and phase of ultrashort light pulses from spectrally resolved autocorrelation. Opt. Lett. 18: 1946-1948

Salin F, Georges P, Roger G, Brun A 1987 Single-shot measurement of a 52-fs pulse. Appl. Opt. 26: 4528-4531

Strickland D, Mourou G 1985 Compression of amplified chirped optical pulses. Opt. Commun. 56: 219-221

Vora H S, Nakhe S V, Sharangpani K K, Saxena P, Bhatnagar R, Shirke N D 1994 Profile monitor for laser beam parameter measurements. Proc. National Laser Symposium, Centre for Advanced Technology, Indore

Walmsley I A, Wong V 1996 Characterization of the electric field of ultrashort optical pulses. J. Opt. Soc. Am. B11: 2453-2463 